\title{
The Estimation of the Time Constant of the Human Inner Ear Pressure Change by Noninvasive Technique
}

\author{
Raghida Traboulsi, ${ }^{1,2}$ Georges Poumarat, ${ }^{1}$ Jean Chazal, ${ }^{2}$ Paul Avan, ${ }^{2}$ Thierry Mom, ${ }^{2}$ \\ Isabelle Ranchon-Cole, ${ }^{2}$ and Salam Traboulsi ${ }^{2}$ \\ ${ }^{1}$ Laboratory of Biomechanics and Anatomy, School of Medicine, Clermont 1 University, BAPS EA 3533, Clermont-Ferrand, France \\ ${ }^{2}$ Laboratory of Sensory Biophysics, School of Medicine, Clermont 1 University, Clermont-Ferrand, France
}

Correspondence should be addressed to Raghida Traboulsi, rtraboulsi@yahoo.fr

Received 18 August 2008; Accepted 22 April 2009

Recommended by Andrzej Swierniak

\begin{abstract}
We propose a noninvasive method to estimate the time constant. The calculation of this factor permits us to understand the pressure variations of the inner ear and also predict the behavior of the flow resistance of the cochlear aqueduct. A set of mathematical relationships incorporating the intralabyrinthine pressure, the intracranial pressure, and the time constant was applied. The modeling process describes the hydrodynamic effects of the cerebrospinal fluid in the intralabyrinthine fluid space, where the input and output of the created model are, respectively, the sinusoidal variation of the respiration signal and the distortion product of otoacoustic emissions. The obtained results were compared with those obtained by different invasive techniques. A long time constant was detected each time when the intracranial pressure increased; this phenomenon is related to the role of the cochlear aqueduct described elsewhere. The interpretation of this model has revealed the ability of these predictions to provide a greater precision for hydrodynamic variation of the inner ear, consequently the variation of the dynamic process of the cerebrospinal fluid.
\end{abstract}

Copyright ( $) 2009$ Raghida Traboulsi et al. This is an open access article distributed under the Creative Commons Attribution License, which permits unrestricted use, distribution, and reproduction in any medium, provided the original work is properly cited.

\section{Introduction}

The intracranial pressure (ICP) has been shown to influence the perilymphatic pressure [1-3], because the cranial cerebrospinal fluid (CSF) and inner ear intralabyrinthine fluid communicate through the cochlear aqueduct [4-8]. The ICP changes on a second-to-second basis [9]. Superimposed on the baseline ICP are periodic changes due to cardiovascular activity and respiration $[4,10,11]$. In addition, the ICP varies with the posture change $[12,13]$. To achieve a new equilibrium state between the hydrodynamical interactions, a variation of the flow resistance $\left(R_{A}\right)$ of the cochlear aqueduct has been linked to the perpetual state of flux $[2,14,15]$. The cochlear aqueduct would be a lowpass filter that should be able to transmit infrasonic waves (i.e., below of $20 \mathrm{~Hz}$ ) from CSF to the cochlea [6].

Densert et al. have investigated the inner ear pressure by measuring a time constant for pressure release [16-18]. This time constant reflects the pressure change of the inner ear.
The experiment was carried out by perforating the tympanic membrane of the cat, applying a square wave pressure at a low frequency of $6.25 \mathrm{mHz}$, and measuring the time constant by introducing a sound in the perilymphatic fluid. Each pressure applied to the middle ear resulted in a double response in the perilymphatic fluid. The first time constant was referred to as the initial response " $\tau_{1}$ ", and it appeared instantaneously upon the application of the stimulus. The second response " $\tau_{2}$ " was a direct reaction to the cessation of the pressure stimulus. Later, Wit et al. repeated a similar experiment on the guinea pig and calculated a new time constant called $\left(R_{A} C\right)$ [19-21]. $R_{A} C$ was mainly determined by the flow resistance of the aqueduct, combined with the compliance of the cochlear windows. The characteristic function of $R_{A}$ was deduced from a relationship between the intracranial pressure ICP, intralabyrinthine pressure ILP, and time constant $R_{A} C$. However, because both of these techniques are invasive and use a frequency of $6.25 \mathrm{mHz}$ that belongs to the frequencies band diseases, they cannot 
be used to measure the time constant and so to characterize the resistance of cochlear aqueduct in normal or pathological conditions in humans.

In this context, the aim of our work was to estimate under hydrodynamic conditions, the variation of the time constant $R_{A} C$ in the human inner ear with a noninvasive technique. In this mathematical model, the estimated time constant reflects the necessary duration of the cochlear aqueduct to reach an equilibrium state between the intracranial and the intralabyrinthine spaces. This idea is based on recent experiments of Büki et al. in 2000 [22]. These authors have shown that distortion product of the otoacoustic emissions (DPOAE) generated around $1 \mathrm{kHz}$ respond to pressurerelated stapes impedance changes with change in phase relative to the generator tones, and provide a noninvasive means of assessing intralabyrinthine pressure changes [23]. They also demonstrated from their protocol the absence of any significant confounding middle-ear [24] effect to intracranial pressure ICP. They were described [25] as the relationship between intralabyrinthine and cerebrospinal fluid pressure from the otoacoustic emissions (OAEs) techniques.

Our technique is based on the transmission of infrasonic pressure waves from cerebrospinal to intralabyrinthine fluids through the human cochlear aqueduct [26], by utilizing the recording of the DPOAE stimulus. The estimated $R_{A} C$ values were characterized as a function of the dynamic modulation between intralabyrinthine and intracranial pressures, and then their mean values as a function of the posture body were deduced.

\section{Materials and Method}

2.1. Subjects. All experiments were performed on young, healthy volunteers with normal-hearing of both genders ( 4 males, 4 females, age ranging from 22 to 32 ). Volunteers were instructed not to swallow, to keep reasonably quiet and breathe naturally. They were placed on a tilting table enabling three postures: up-right, supine flat on their back on a horizontal plane, and finally head down $-20^{\circ}$ with respect to horizontal plane.

\subsection{Time Constant Estimation Method}

2.2.1. Data Acquisition. To estimate the time constant $R_{A} C$ of the inner ear, DPOAE, and thoracic signals, 250 to 450 points were recorded over 2-3 minutes with a sampling frequency equal to $6.25 \mathrm{~Hz}$, as already previously described [26]. Briefly, DPOAEs are recorded at about $1 \mathrm{kHz}$ after stimulation of two tones of frequency $f_{1}$ and $f_{2}$ where $f_{2} / f_{1}$ ratio is equal to 1.2. The corresponding primary levels $L_{1}$ and $L_{2}$ of DPOAE were set at $70 \mathrm{~dB}$ SPL in the sealed ear canal. The DPOAE and thoracic signals data (Figure 1) are then filtered to obtain nonlinear smoothing signals and to select the infrasonic waves of the ICP and ILP, corresponding to the respiratory frequency band between 0.17 and $0.5 \mathrm{~Hz}$. These methods have been shown to be effective in removing noise and artifacts from DPOAE without blurring eventual physiological modulations. Thereafter, the normalized ICP

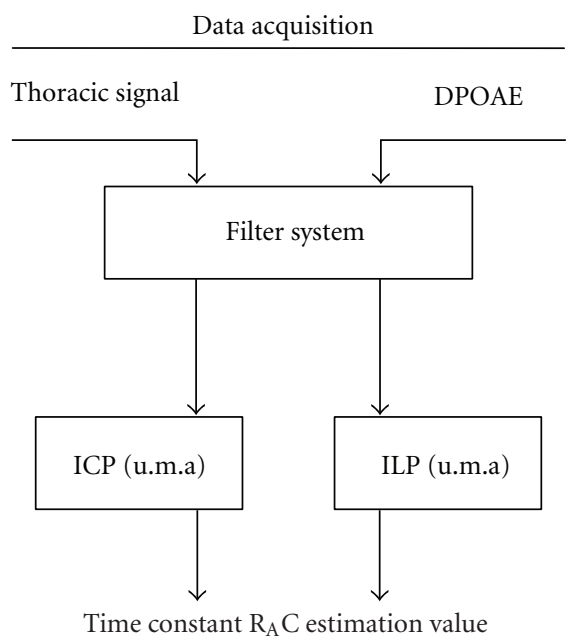

Figure 1: The following diagram shows the methodology of how the time constant is estimated. The infrasonic waves of the ICP and ILP were filtered from the thoracic and DPOAE signals; each infrasonic wave was between $(0.166 \mathrm{~Hz}, 0.5 \mathrm{~Hz})$, noticed that the reference [26] gives more details about the filter system.

and ILP infrasonic waves were used as the input and output signals, respectively, for the model system.

2.2.2. Model System. Based on the work of Gopen et al. [6] and Wit et al. [21] on the inner ear, a simplified mechanical model was created (Figure 2(a)). This model describes the connections and interconnections between the three different compartments of the human body: the respiratory $\left(C_{1}\right)$, the head $\left(C_{2}\right)$, and the auditory systems $\left(C_{3}\right)$. Thereafter, the equivalent electrical parameters to this model (Figure 2(b)): CSF characteristics are represented by the lumpedelement Rcsf, Lcsf, and Cc [26], the cochlear aqueduct connecting the cochlea to these spaces acts as a resistance $R_{A}$. The cochlear pressure signal transmitted from the CSF spaces is applied both at the round window and stapes but their large impedance difference implies that only the round window and its compliance $C$ can play a significant part.

This model has been created for two objectives. The first one was to calculate the input signal which defines the relationship between the thoracic signal and the cranial fluid pressure. The second was to analytically prove from the output signal, the relationship between the variations of the cochlear response with the movement of the cerebrospinal fluid (CSF).

2.2.3. Input Signal. The input signal is represented by $P_{c}$ which is the dynamic intracranial pressure ICP related to the rapid fluctuation of breathing. To calculate $P_{c}$, we started by modeling the thoracic impedance $Z(t)$ with the blood variation in the chest. If the imposed changes in pressure and resulting flow are sufficiently small, $Z(t)$ is represented in terms of a single resistance and single compliance [27] as

$$
Z(t)=Z_{m} \sin (\omega t+\alpha)
$$


where $Z_{m}$ is the maximum chest impedance, $\omega$ represents the respiratory variation and is equal to $2 \pi f[1 / \mathrm{s}]$ with $f$ varying between $[0.17,0.5] \mathrm{Hz}$ and $\alpha$ is a constant.

$Z(t)$ is also defined by the total impedance of the blood and tissue longitudinal impedances given [28] by

$$
Z(t)=\frac{Z_{b} \times Z_{t}}{\left[Z_{b}+Z_{t}\right]},
$$

where $Z_{b}$ and $Z_{t}$ are the blood and tissue longitudinal impedance, respectively, and are expressed in $\left[\mathrm{kg} \times \mathrm{m}^{4}\right]$.

At the same time, it is known that

$$
d V_{b}=d\left(l A_{b}\right)=-\left[\frac{\left(\rho_{b} l^{2}\right)}{Z_{b}^{2}}\right] d Z_{b},
$$

where " $d V_{b}$ " is the variation blood volume in the chest, " $A_{b}$ " expressed in square meters is the cylindrical base area, "l" expressed in meters is the chest length, and " $\rho_{b}$ " $\left[\mathrm{kg} / \mathrm{m}^{3}\right]$ is the resistivity of the blood [28].

Because the respiratory and cardiac movements are transported by the blood to the cerebrospinal fluid [11] in (3), $Z_{b}$ can be modeled by the sinusoidal variation of $Z(t)$.

The blood pressure variation $d P[\mathrm{~Pa}]$ in the chest, is related to the compliance factor $C_{b}\left[\mathrm{~m}^{3} / \mathrm{Pa}\right]$ and to the variation of the respiration volume [27] by the following relation:

$$
C_{b}=\frac{d V}{d P} .
$$

If we consider that, the connection between the respiratory system and the brain has a laminar flow, that is, like a shortcircuit (or like a resistance with constant value), then $d P$ values can be defined as the input signal of the electrical circuit in figure (Figure 2(b)). From (3) and (4) the variation of blood pressure with the impedance $Z_{b}$

$$
d P=-\left[\frac{\left(\rho_{b} l^{2}\right)}{C_{b} Z_{b}^{2}}\right] d Z_{b}
$$

was obtained.

This differential equation can be considered as a definite integral with $d P$ varying between $\left[0, P_{c}\right]$ and $d Z_{b}$ between $\left[Z_{0}, Z_{m}\right]$. Therefore, the equation of the input signal is as follows:

$$
\begin{aligned}
P_{c} & =\left[\frac{\left(\rho_{b} l^{2}\right)}{C_{b} Z_{m}}\right] \cos (\omega t+\alpha) \\
& =P_{m} \cos (\omega t+\alpha),
\end{aligned}
$$

where $P_{c}$ is the dynamic intracranial pressure ICP related to the rapid fluctuation of breathing in the cerebrospinal fluid CSF. $P_{m}$ is the maximal amplitude of $P_{c}$.

Equation (6) is a simplified model of $P_{c}$; this equation is valid and may represent the movement of the rapid fluctuation linked to the respiratory system.

Validation of the Input Signal Equation. The resulting amplitude $P_{m}, P_{m}=\left[\left(\rho_{b} l^{2}\right) / C_{b} Z_{m}\right]$ demonstrates that the influence of the respiration on CSF flow is decreased in cranial direction [11], and if we calculate the unit of $P_{m}$,

$$
\begin{aligned}
{\left[P_{m}\right] } & =\left(\frac{\left[\rho_{b}\right] \times[l]^{2}}{\left[C_{b}\right] \times\left[Z_{m}\right]}\right) \\
& =\left(\frac{[\mathrm{kg}] \times\left[\mathrm{m}^{-1}\right]}{\left[\mathrm{m}^{3}\right] \times\left[\mathrm{Pa}^{-1}\right] \times[\mathrm{kg}] \times\left[\mathrm{m}^{-4}\right]}\right) \\
& =[\mathrm{Pa}] .
\end{aligned}
$$

2.2.4. Output Signal. The intralabyrinthine pressure ILP in the inner ear is considered as the output signal (Figures $2(\mathrm{a})$ and 2(b)), this output signal is represented by $P_{i}$. Our objective was to find the relationship between ILP, ICP, and $R_{A} C$. Based on the physical model described by Feijen et al. [20], the differential equation

$$
\left(R_{A} C\right)\left(\frac{d P_{i}}{d t}\right)=P_{i}(t)-P_{c}(t)
$$

was resolved, but rather than sending a square signal, a sinusoidal signal of $P_{c}(t)$ was injected (6), therefore we obtained:

$$
P_{i}(t)=S_{1} \times\left[\cos (\omega t+\alpha)+S_{2} \times \sin (\omega t+\alpha)\right],
$$

where

$$
S_{1}=\frac{P_{m}}{\left[1+\left(R_{A} C \omega\right)^{2}\right]}
$$

is the maximal amplitude pressure in $[\mathrm{Pa}]$ of intralabyrinthine fluid in the cochlea at the time constant $\tau=R_{A} C$ in [s] and $S_{2}=R_{A} C \omega, S_{1}$, and $S_{2}$ vary with the variation of the respiration frequency $\omega[1 / \mathrm{s}]$.

We can deduce the relationship between the intracranial and the intralabyrinthine pressure by substituting (6) in (9):

$$
P_{i}(t)+\left(R_{A} C \omega\right)^{2} P_{i}(t)=P_{c}(t)+P_{m}\left(R_{A} C \omega\right) \sin (\omega t+\alpha) .
$$

As we can see in (11), the relationship between the two factors is strongly related to the frequency of respiration $\omega$ and to the time constant of the inner ear.

By replacing $\sin (\omega t+\alpha)$ by $\left(1-\cos ^{2}(\omega t+\alpha)\right)^{1 / 2}$, a new relationship between ICP and ILP was deduced. The following relationship was obtained:

$$
S_{2}^{2} \times\left|P_{i}(t)\right|+S_{2} \times\left(\left|P_{m}^{2}-P_{c}(t)^{2}\right|\right)^{1 / 2}+\left|P_{i}(t)-P_{c}(t)\right|=0,
$$

where $S_{2}$ is $R_{A} C \times \omega=\tau \times \omega, P_{m}$ is the maximum intracranial pressure, $P_{i}(t)$ is the intralabyrinthine pressure (ILP) or the cochlear response at instant " $t$ ", and $P_{c}(t)$ is the dynamic intracranial pressure (ICP) represented by the rapid fluctuation of the breathing at instant " $t$ ". Considering $\omega$ as a constant at a given frequency of respiration. The time constant $\tau=\left(R_{A} C\right)$ has a role of independent variable, where his positivity is necessary for a valid estimate. The $\left|P_{i}(t)-P_{c}(t)\right|$ is the constant parameter at " $t$ " second. For each selected $\left|P_{i}(t)-P_{c}(t)\right|$ changes. 


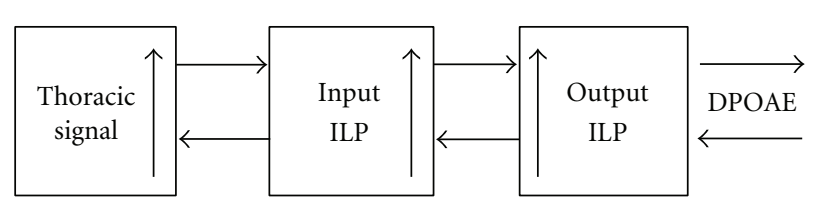

(a)

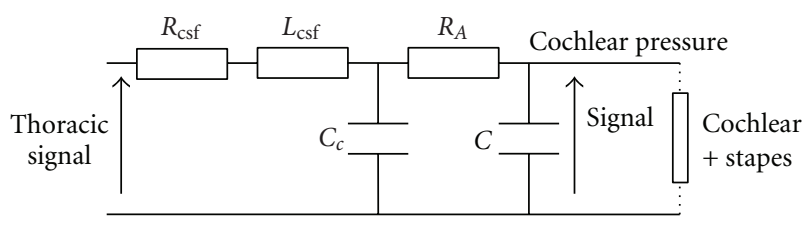

(b)

Figure 2: Model system. (a) Mechanical system. (b) Equivalent electrical circuit of the model.

\section{Results}

To estimate the variation of the time constant $R_{A} C$, the infrasonic waves (between 0.17 and $0.5 \mathrm{~Hz}$ ) of ICP and ILP from DPOAE and respiration databases were extracted. ICP and ILP are represented by $P_{c}(t)$ and $P_{i}(t)$, respectively (see also paragraph §.2). A database for each subject for 3 different postures was recorded separately: up-right $\left(90^{\circ}\right)$, supine flat on their back on a horizontal plane $\left(0^{\circ}\right)$, and finally head down $\left(-20^{\circ}\right)$ with respect to horizontal plane. Figure 3 gives a representative example of a database for one posture. The recordings of dynamic pressure modulation $\left(P_{i}(t)-P_{c}(t)\right)$ and $P_{i}(t)$ are sinusoidal signals. Each recording can be divided in two different parts depending on the physiological condition. Indeed, the two sinusoidal curves $\left(P_{i}(t)-P_{c}(t)\right)$ and $P_{i}(t)$ of Figure 3 are:

(1) vibrating very differently which we called the transition signal part; this turbulent transition process was observed each time after a variation of the respiration frequency $(\omega=2 \pi f)$, or sometimes, because of the physiological changes that are still unknown,

(2) when they are both on the same phase.

Thereafter, using (12), we estimated $R_{A} C$ for each sequence of each recording obtained for each subject and each posture. A sequence (or wavelength) is composed of two peaks representing the consecutive positive and negative pressures (Figure 4).

Each peak is divided into an up-going edge and a downgoing edge. The resulting solution was found to contain two time constants variables $R_{A} C\left(\tau_{1}\right.$ and $\left.\tau_{2}\right)$. Because $\tau_{2}$ was significantly smaller than $\tau_{1}$, we only represent $\tau_{1}$. The edges varying between 6 and 7 points, we obtained 6 to $7 R_{A} C$ values. We observed that $R_{A} C$ was giving a uniform curve variation for the sequences contained in the same phase signal part; therefore for all the results presented thereafter, we discarded the perturbation signal part.

3.1. $\mathbf{R}_{\mathbf{A}} \mathbf{C}$ as a Function of the Dynamic Pressure Modulation. $R_{A} C$ was estimated for each sequence of the same phase signal part. Then, $R_{A} C$ was plotted as a function of $\mid P_{i}(t)-$ $P_{c}(t) \mid$ for the positive and for the negative pressures (Figures 5(a) and 5(b), resp.). $R_{A} C$ followed a uniform and regular pattern for all sequences located without the turbulent transition. For the positive pressure, $R_{A} C$ progressively increased on the up-going edge, until it reached a maximum corresponding to the maximum of the $\left|P_{i}(t)-P_{c}(t)\right|$ and

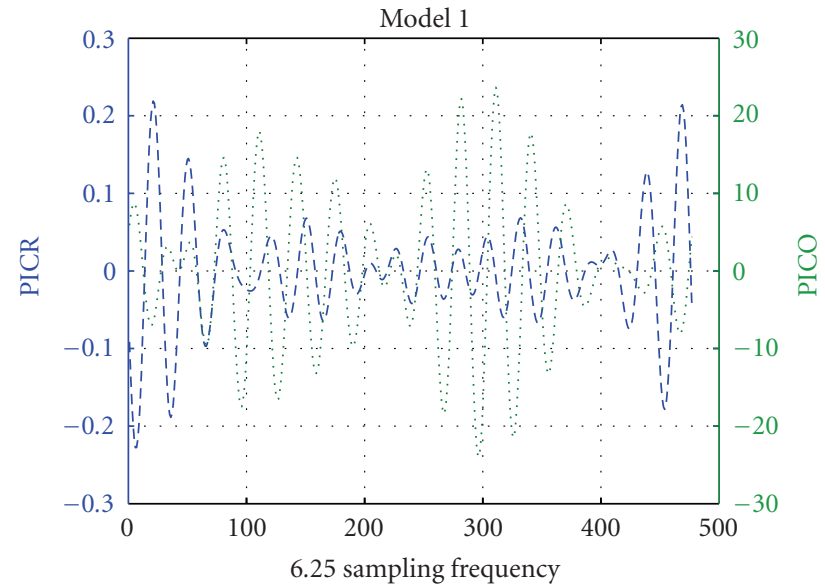

FIGURE 3: $P_{i}(t)$ in broken line is the intralabyrinthine inner ear pressure (ILP) or the infrasonic response of the cochlea, and the second curve $P_{i}(t)-P_{c}(t)$ is the modulation of the cochlear signal versus the cerebral fluctuation. We observed a perturbation status in the first 70 points, then after 400 points, these points are rejected from our estimation.

$P_{i}(t)$, and then it progressively decreased on the downgoing edge. For the negative pressure, it is the opposite: on the down-going edge, $R_{A} C$ increased until it reached its maximum which corresponds to the minimum of $\left(P_{i}(t)-\right.$ $\left.P_{c}(t)\right)$ and $P_{i}(t)$, while it decreased on the up-going edge. For a given $\left|P_{i}(t)-P_{c}(t)\right|$ magnitude, $R_{A} C$ is not the same in the negative or positive edge. For all subjects, $R_{A} C$ does not vary symmetrically in the positive and negative pressures (see, e.g., $P_{i}(t)-P_{c}(t)$ at 0.4 and -0.4 of Figures $5(\mathrm{a})$ and $\left.5(\mathrm{~b})\right)$. Therefore, these results show that to increase the modulation of dynamic pressure $\left|P_{i}(t)-P_{c}(t)\right|$ leads to an increase in the cochlear aqueduct resistance.

3.2. $\mathbf{R}_{\mathbf{A}} \mathbf{C}$ as a Function of Body Posture. $R_{A} C$ was estimated for each sequence in the same phase signal part. The $12-14 R_{A} C$ values obtained for the positive or negative pressures of one sequence were averaged to obtain a mean $R_{A} C$ value. Then, the mean $R_{A} C$ values were plotted as a function of the body posture: up-right, supine flat and headdown at $-20^{\circ}$ with respect to horizontal plane (Figures 6(a) and $6(\mathrm{~b}))$.

The mean $R_{A} C$ value is not always going ascendancy during the transition from up-right to supine flat, or from supine flat to head-down and does not vary linearly. As one began to move down subject's head from up-right position 


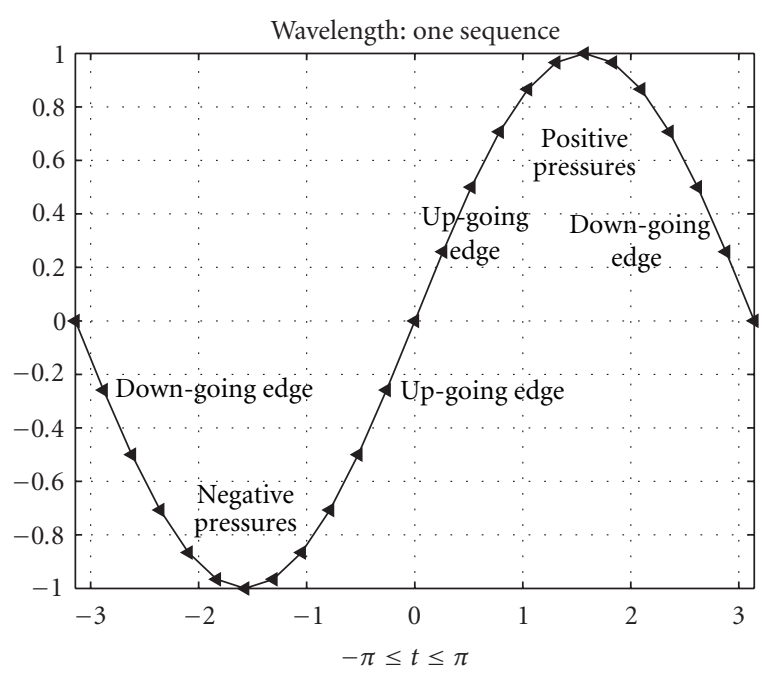

FIGURE 4: We show here the partition of one sequence of sinusoidal curve; this sequence can be a wavelength of intralabyrinthine pressure $P_{i}(t)$, intracranial pressure $P_{c}(t)$, or modulation pressures between $P_{i}(t)$ and $P_{c}(t)$.

to horizontal plane $0^{\circ}$, there was always a variation of $R_{A} C$ value, the mean $R_{A} C$ can continue to increase or decrease according to each person. With head-down tilting at $-20^{\circ}$, mean $R_{A} C$ has continued to increase except for two persons (2) and (5) of Figures 6(a) and 6(b), but its value has always remained with incremental changes being much greater than from head-up tilting. For a given posture, the mean $R_{A} C$ varies from one subject to the other. However, the variation range is similar in the positive and negative pressure. Indeed, on the up-right posture, mean $R_{A} C$ varies from 1.2 to 17.1 in the positive pressure and from 1.6 to 22.8 in the negative pressure; on the horizontal posture, mean $R_{A} C$ varies from 1.3 to 24.0 in the positive pressure and from 1.6 to 34.9 in the negative pressure; on the head-down tilting at $-20^{\circ}$, mean $R_{A} C$ varies from 6.0 to 42.4 in the positive pressure and from 7.5 to 56.6 in the negative pressure. We can deduce from these mean values, the maximum variation range was provided in the head-down posture at $-20^{\circ}$, being 36.4 for the positive pressure and 49.1 for the negative pressure.

\section{Discussions}

Intralabyrinthine pressure monitoring in humans is potentially interesting in two situations [29]: hydrocephalus (with abnormal ICP) and Menière's disease (with an alleged pathology of endolymphatic hydrostatics). Using invasive measurements is difficult to perform because of the vulnerability of the human inner ear. The changes in OAE levels in ears with Menière's disease have been proposed as being potentially useful indicators, for clinical monitoring of labyrinth function [29-31]. The DPOAE signals provide an original way to estimate the time constant by noninvasive technique.

Very long time constants were observed by Densert et al. [16] after the blockage of the cochlear aqueduct. In our results, the $R_{A} C$ reaches its maximum value relating mainly to overpressure and under-pressure. The positive peak values of the modulation $\left|P_{i}(t)-P_{c}(t)\right|$ indicates an over pressure, while the negative peak is the under-pressure. The modulation $\left|P_{i}(t)-P_{c}(t)\right|$ also provides pressure at the cochlear aqueduct. The monitoring and processing of pressure states ILP and ICP, indicates the flow parameters in a fluid assembly; the information of the cochlear aqueduct [20] is provided by this equation: $R_{A}=\left(P_{i}-P_{c}\right) / f$, where $f$ is the fluid flow between intralabyrinthine and cerebral space in $\left[\mathrm{m}^{3} / \mathrm{s}\right]$. The phenomenon of long-time constants $R_{A} C$, detected in our results at peak $\left|P_{i}(t)-P_{c}(t)\right|$, was explained from this mathematical relationship by the increasing value of the resistance $R_{A}$ of the cochlear aqueduct, or decreasing of the fluid flow, where the cochlear aqueduct plays a prominent role and inhibits a large amount of fluid between the two spaces $[1,6,14]$.

Knowing that $P_{i}(t)$ is the inner ear pressure, it presents the global variation pressures including the endolymphatic system, perineural and perivascular spaces. The $P_{i}(t)$ magnitude on the area without turbulent transition in our databases (Figure 3), was almost equal to the modulation magnitude of $\left(P_{i}(t)-P_{c}(t)\right), P_{i}(t)$ phase was proportionally varied with the $\left(P_{i}(t)-P_{c}(t)\right)$ of the cochlear aqueduct pressures. Therefore the change in the time constant can be explained by the change of the flow resistance [2] during inner ear pressure variation provided by the permeability change of the cochlear aqueduct, caused by a change of structures filling the aqueduct and its entrance in scala tympani. Nevertheless, the regulation of the physiological variations of the inner ear pressures appears to be well balanced even in individuals with poor patency of the cochlear aqueduct, probably due to the close hydrodynamic relationship between the endolymphatic and the cerebrospinal fluid systems [32]. On the other hand, during the turbulent transition indicated on Figure 3 , the $P_{i}(t)$ was inferior and nonproportionally varied with the $\left(P_{i}(t)-P_{c}(t)\right)$ of the cochlear aqueduct pressures. Therefore the change of the time constant is characterized by fast nonlinear curves and generally by variability of one sequence to another. This can be explained by the role of the cochlear aqueduct [4-7]. The cochlear aqueduct quickly modifies its flow resistance; because it acts as a lowpass filter to ICP changes and attenuates frequency components above its cutoff frequency, where pressure equalization caused by the different factors takes place within seconds.

We can quantify the characteristic of $R_{A}$, if we divide the obtained $R_{A} C$ by the window compliance $C$ [21]. As we know $C$ is defined by the ratio of window pressure and volume for the human (round + oval) window. Ivarsson and Pedersen demonstrated the relationship between the window pressure and this compliance $C$ [33]. This relationship demonstrates that, $C$ only changes a few percentage points for the range of window pressure variation during an experiment. Consequently, the characteristic behavior of the flow resistance of the cochlear aqueduct can be similar to those obtained from $R_{A} C$.

Comparisons were made between our curves (Figures 6(a) and 6(b)) with those obtained by Chapman et al. [13], 


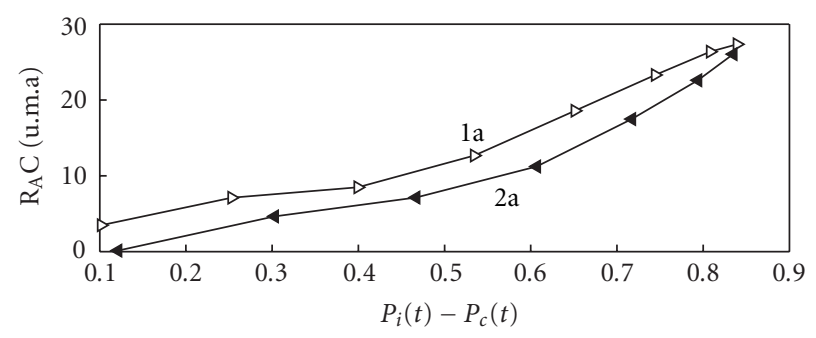

(a)

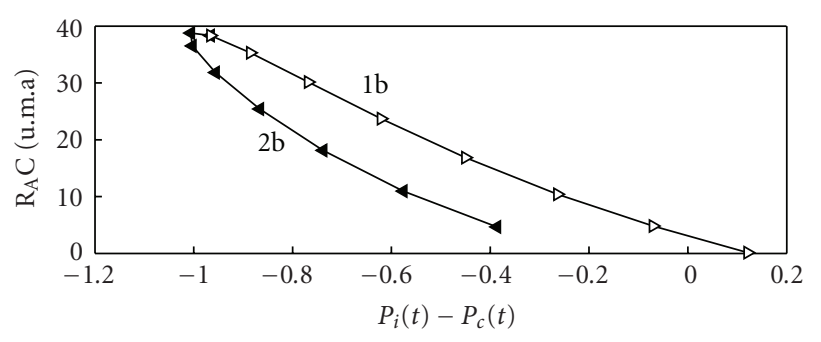

(b)

Figure 5: The vertical axis of (a) and (b) is the time constant values, and the horizontal axis is the sinusoidal modulation $\left(P_{i}(t)-P_{c}(t)\right)$. The first part " $1 \mathrm{a}$ " at the top on the resulting curves in (a) is the $R_{A} C$ values calculated from the positive up-going points of $\left(P_{i}(t)-P_{c}(t)\right)$ and $P_{i}(t)$, "2a" obtained from the positive down-going. In (b), " $1 \mathrm{~b}$ " is obtained from the down-going points of $\left(P_{i}(t)-P_{c}(t)\right)$ and $P_{i}(t)$, and "2b" from negative up-going pressures. Notice that (a) and (b) are, respectively, Figures 5(a) and 5(b).

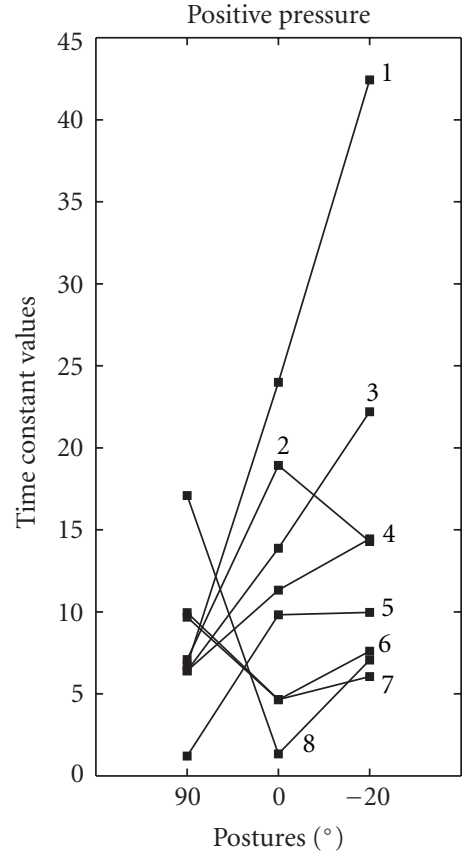

(a)

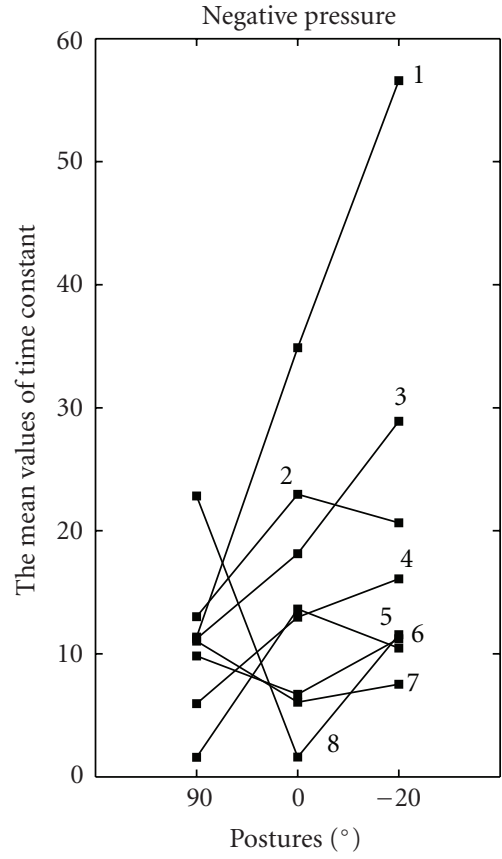

(b)

Figure 6: The mean values of time constant variation $R_{A} C$ in the positive pressure edges (a) and negative pressure edges (b), in 8 ears, according to three different postures : up-right $\left(90^{\circ}\right)$, supine flat on their back on a horizontal plane $\left(0^{\circ}\right)$, and head down $\left(-20^{\circ}\right)$ with respect to horizontal plane. Noticed that (a) and (b) are, respectively, Figures 6(a) and 6(b).

and are similar. The author has determined, by invasive technique, the quantitative relationship between changes in body position and ventricular fluid pressure (intracranial pressure) in normal subjects, using a chronically implanted telemetric pressure sensor. A comparison between this invasive technique and our noninvasive estimation, has been possible because on the normal subject the inner ear fluid pressure can be suddenly changed by the interaction between the intracranial and intralabyrinthine fluids in the normal labyrinth, consequently the inner ear can mirror the variation of the intracranial pressures [15]; adding also, without the normal condition, many other factors can disturb the regulation pressure of the inner ear, such as a change of ear canal [34], middle ear pressure [18] or injection of fluid [19].
Studies in guinea pigs have shown a relation between acute inner ear pressure changes and cochlear function at low-level DPOAE. The inner ear pressure was represented by the variation of the time constant [3]. The authors studied the amplitude and phase of the DPOAE and showed the effect of the DPOAE stimulus and inner ear pressure changes, during and after injection of $0.5 \mu \mathrm{L}$ of artificial perilymph in the inner ear. The two primary frequencies were set at $6 \mathrm{kHz}$ $\left(f_{1}\right)$ and $7.5 \mathrm{kHz}\left(f_{2}\right)$ with $f_{1} / f_{2}$ ratio $=1.5$, with intensities set, respectively, at $65 \mathrm{~dB} \operatorname{SPL}\left(L_{1}\right)$ and $55 \mathrm{~dB} \operatorname{SPL}\left(L_{2}\right)$. These high frequencies are used to provide a DC special condition, where the direct current flow is caused to vary very slightly in a special way, with the tiny variations happening at a very high speed, so as to produce a square wave acoustical signal from the DPOAE stimulus when compared to a conventional 
condition of the input square signal applied by the previous work of Feijen et al. [20]. However, we cannot compare our prediction results to this experiment because we do not have the same frequencies or the same DPOAE stimulus. In our experiment, the DPOAE frequencies have been around $1 \mathrm{kHz}$ and the phase of DPOAE only has been studied. The modeling process of these estimations from our experiment provides a good "apparent" behavior of $R_{A} C$, because the ICP variations were large $(>350 \mathrm{~mm}$ water or $\mathrm{daPa})$, and OAEs seemed little affected above $2 \mathrm{kHz}$. Below $1 \mathrm{kHz}$, their levels tended to decrease by hardly more than $2 \mathrm{~dB}$. The most compicuous change concerned the phase of low-frequency components of OAEs: below $1.8 \mathrm{kHz}$, it tended to lead the reference phase measured in low ICP conditions, and the size of the phase lead was found to be maximum at around $1 \mathrm{kHz}$. The maximum phase lead, averaged across ears, turned out to be proportional to ICP increase, according to the linear regression [23]:

$$
\Delta \text { phase }=0.20 \Delta \mathrm{ICP} \text { (phase degrees, ICP in daPa). }
$$

The main advantage of this estimation is to obtain the time constant of the pressure variation of the inner ear by a noninvasive technique. We believe that, if we take into account this simple predictor provided by the modeling process, it is possible to use the results of infrasonic waves extracted from the DPOAE stimulus around $1 \mathrm{kHz}$ as an aide to predict the hydrodynamic behavior of the cochlear aqueduct below $20 \mathrm{~Hz}$, therefore the relationship of the hydromechanical interactions between the intracranial and intralabyrinthine fluids.

\section{Conclusion}

The modeling process is the only way to understand the hydrodynamical interactions between the intracranial and intralabyrinthine fluids in the inner ear, because it provides a noninvasive measurement. Using the fact that intralabyrinthine pressure changes induce characteristic phase shifts of DPOEAs around $1 \mathrm{kHz}$. The results are consistent with previous estimations of the time constant of the inner ear derived from invasive animal experiments. Noninvasive measurements of the time constant at low-frequency pressure waves may turn out to be applicable to monitoring the normal physiology and pathophysiology of the inner ear. This modeling was applied to healthy subjects, changes in the mathematical equations of this model will be necessary, if we want to estimate the time constant for different pathological conditions.

\section{Acknowledgments}

The authors would like to acknowledge the support of Eric Le Page, Director of OAEricle Laboratory (Australia). They would like to thank the professors of ESITPA for their encouragement for the finalization of this work.

\section{References}

[1] A. Hara, A. N. Salt, and R. Thalmann, "Perilymph composition in scala tympani of the cochlea: influence of cerebrospinal fluid," Hearing Research, vol. 42, no. 2-3, pp. 265-272, 1989.

[2] B. I. R. Carlborg, K. S. Konradsson, A. H. Carlborg, J. C. Farmer Jr., and O. Densert, "Pressure transfer between the perilymph and the cerebrospinal fluid compartments in cats," The American Journal of Otology, vol. 13, no. 1, pp. 41-48, 1992.

[3] W. L. Valk, H. P. Wit, and F. W. J. Albers, "Effect of acute inner ear pressure changes on low-level distortion product otoacoustic emissions in the guinea pig," Acta OtoLaryngologica, vol. 124, no. 8, pp. 929-936, 2004.

[4] R. J. Marchbanks and A. Reid, "Cochlear and cerebrospinal fluid pressure: their inter-relationship and control mechanisms," British Journal of Audiology, vol. 24, no. 3, pp. 179-187, 1990.

[5] A. Böhmer, "Hydrostatic pressure in the inner ear fluid compartments and its effects on inner ear function," Acta OtoLaryngologica, no. 507, pp. 3-24, 1993.

[6] Q. Gopen, J. J. Rosowski, and S. N. Merchant, "Anatomy of the normal human cochlear aqueduct with functional implications," Hearing Research, vol. 107, no. 1-2, pp. 9-22, 1997.

[7] E. O. Thalen, H. P. Wit, J. M. Segenhout, and F. W. J. Albers, "Dynamics of inner ear pressure change caused by intracranial pressure manipulation in the guinea pig," Acta Oto-Laryngologica, vol. 121, no. 4, pp. 470-476, 2001.

[8] T. Nakashima, S. Naganawa, M. Sone, et al., "Disorders of cochlear blood flow," Brain Research Reviews, vol. 43, no. 1, pp. 17-28, 2003.

[9] N. Lundberg, "Continuous recording and control of ventricular fluid pressure in neurosurgical practice. Rapid variations of the ventricular fluid pressure," Acta Psychiatric Neurology, vol. 36, pp. 81-177, 1960.

[10] Z. Štěpáník, A. Kaczmarská, J. Otáhal, and S. Otáhal, "Simulation of cardiac and respiration related pulsation in CSF transport," Journal of Biomechanics, vol. 40, article S2, 2006.

[11] J. Otáhal, Z. Štěpáník, A. Kaczmarská, F. Maršík, Z. Brož, and S. Otáhal, "Simulation of cerebrospinal fluid transport," Advances in Engineering Software, vol. 38, no. 11-12, pp. 802809, 2007.

[12] J. Loman, A. Myerson, and D. Goldman, "Effects of alterations in postures on the cerebrospinal fluid pressure," Archives of Neurology \& Psychiatry, vol. 33, pp. 1279-1295, 1935.

[13] P. H. Chapman, E. R. Cosman, and M. A. Arnold, "The relationship between ventricular fluid pressure and body position in normal subjects and subjects with shunts: a telemetric study," Neurosurgery, vol. 26, no. 2, pp. 181-189, 1990.

[14] B. I. Beentjes, "The cochlear aqueduct and the pressure of cerebrospinal and endolabyrinthine fluids," Acta OtoLaryngologica, vol. 73, no. 2, pp. 112-120, 1972.

[15] R. J. Marchbanks, "Hydrodynamical interactions of the intracranial and intralabyrinthine fluids," in Handbook of Intracranial and Intralabyrinthine Fluids: Basic Aspects and Clinical Applications, A. Ernest, et al., Ed., Springer, Berlin, Germany, 1995.

[16] B. Densert, O. Densert, B. Erlandsson, and H. Sheppard, "Transmission of square wave pressure pulses through the perilymphatic fluid in cats," Acta Oto-Laryngologica, vol. 102, no. 3-4, pp. 186-193, 1986. 
[17] B. Densert, O. Densert, S. Arlinger, K. Sass, and L. Ödkvist, "Immediate effects of middle ear pressure changes on the electrocochleographic recordings in patients with Meniere's disease: a clinical placebo-controlled study," American Journal of Otology, vol. 18, no. 6, pp. 726-733, 1997.

[18] O. Densert, B. Carlborg, and J. Stagg, "Transmission of low frequency pressure steps to the perilymphatic fluid," Acta OtoLaryngologica, vol. 91, no. 1-2, pp. 55-64, 1981.

[19] H. P. Wit, E. O. Thalen, and F. W. J. Albers, "Dynamics of inner ear pressure release, measured with a double-barreled micropipette in the guinea pig," Hearing Research, vol. 132, no. 1-2, pp. 131-139, 1999.

[20] R. A. Feijen, J. M. Segenhout, F. W. J. Albers, and H. P. Wit, "Change of guinea pig inner ear pressure by square wave middle ear cavity pressure variation," Acta Oto-Laryngologica, vol. 122, no. 2, pp. 138-145, 2002.

[21] H. P. Wit, R. A. Feijen, and F. W. J. Albers, "Cochlear aqueduct flow resistance is not constant during evoked inner ear pressure change in the guinea pig," Hearing Research, vol. 175, pp. 190-199, 2003.

[22] B. Büki, A. Chomicki, M. Dordain, et al., "Middle-ear influence on otoacoustic emissions. II: contributions of posture and intracranial pressure," Hearing Research, vol. 140, no. 12, pp. 202-211, 2000.

[23] B. Büki, P. Avan, J. J. Lemaire, M. Dordain, J. Chazal, and O. Ribári, "Otoacoustic emissions: a new tool for monitoring intracranial pressure changes through stapes displacements," Hearing Research, vol. 94, no. 1-2, pp. 125-139, 1996.

[24] C. Dai, M. W. Wood, and R. Z. Gan, "Combined effect of fluid and pressure on middle ear function," Hearing Research, vol. 236, no. 1-2, pp. 22-32, 2008.

[25] B. Büki, E. De Kleine, H. P. Wit, and P. Avan, "Detection of intracochlear and intracranial pressure changes with otoacoustic emissions: a gerbil model," Hearing Research, vol. 167, no. 1-2, pp. 180-191, 2002.

[26] R. Traboulsi and P. Avan, "Transmission of infrasonic pressure waves from cerebrospinal to intralabyrinthine fluids through the human cochlear aqueduct: non-invasive measurements with otoacoustic emissions," Hearing Research, vol. 233, no. 12, pp. 30-39, 2007.

[27] F. P. Primiano Jr., Measurements of the Respiratory System. Handbook of Medical Instrumentation, Application and Design, New York, NY, USA, 2nd edition, 1995.

[28] J. Malmivuo and R. Plonsey, Bioelectromagnetism-Principles and Applications of Bioelectric and Biomagnetic Fields, Oxford University Press, New York, NY, USA, 1995.

[29] P. Avan, B. Büki, T. Mom, and J. Chazal, "Otoacooustic emissions and Menière's disease," in Proceedings of the 4th International Symposium on Menière's Disease, pp. 243-246, 1999.

[30] G. K. Martin, L. A. Ohlms, D. J. Franklin, F. P. Harris, and B. L. Lonsbury-Martin, "Distortion product emissions in humans-III: influence of sensorineural hearing loss," Annals of Otology, Rhinology and Laryngology, vol. 99, no. 5, supplement 147, pp. 30-42, 1990.

[31] W. M. Van Huffelen, N. J. M. Mateijsen, and H. P. Wit, "Classification of patients with Meniere's disease using otoacoustic emissions," Audiology and Neuro-Otology, vol. 3, no. 6, pp. 419-430, 1998.

[32] B. I. Carlborg and J. C. Farmer Jr., "Transmission of cerebrospinal fluid pressure via the cochlear aquaduct and endolymphatic sac," American Journal of Otolaryngology, vol. 4, no. 4, pp. 273-282, 1983.
[33] A. Ivarsson and K. Pedersen, "Volume pressure properties of round and oval windows. A quantitative study on human temporal bone," Acta Oto-Laryngologica, vol. 84, no. 1-2, pp. 38-43, 1977.

[34] B. Krukowski, B. Carlborg, and O. Densert, "Nonlinear aspects of infrasonic pressure transfer into the perilymph," Hearing Research, vol. 2, no. 3-4, pp. 207-212, 1980. 

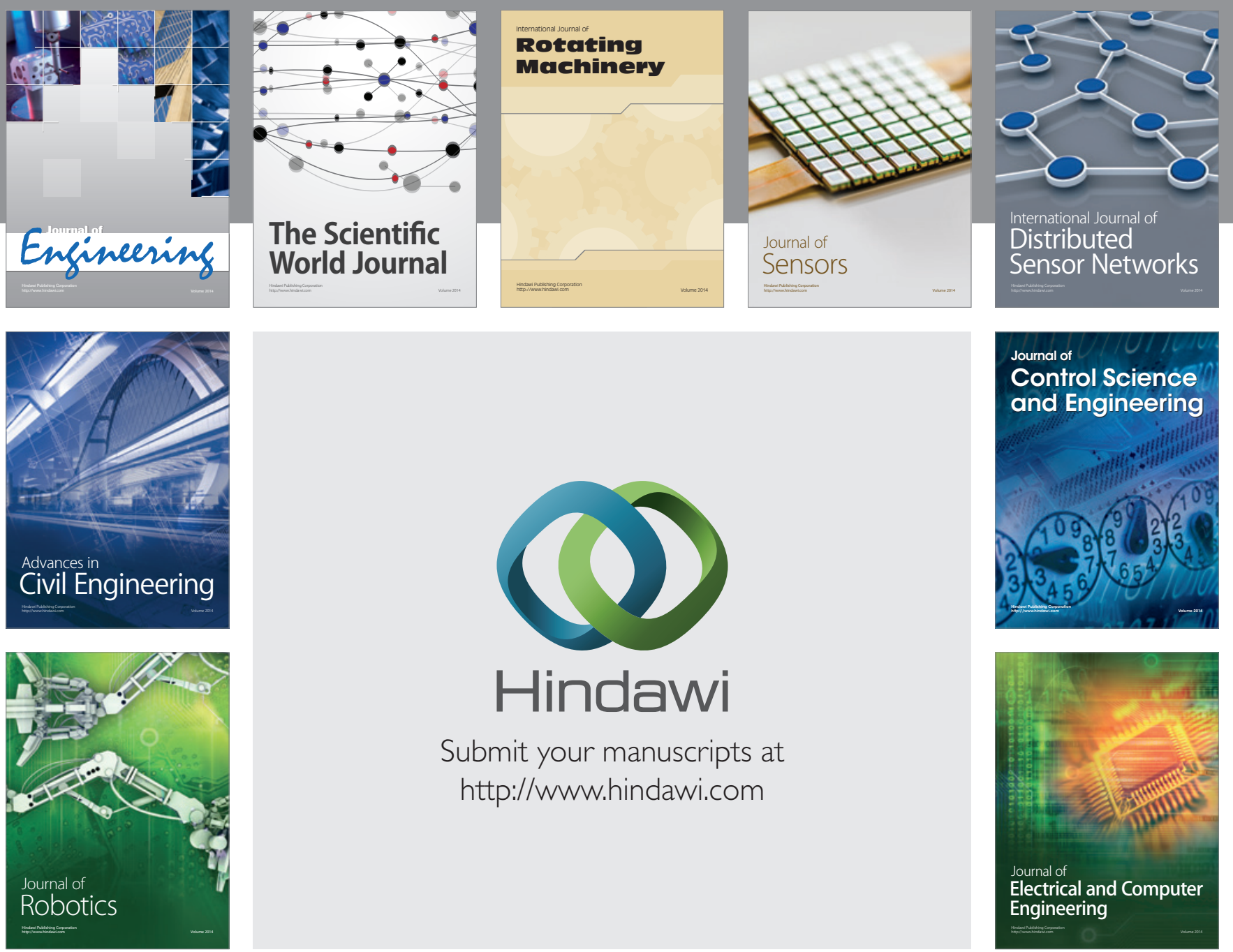

Submit your manuscripts at

http://www.hindawi.com
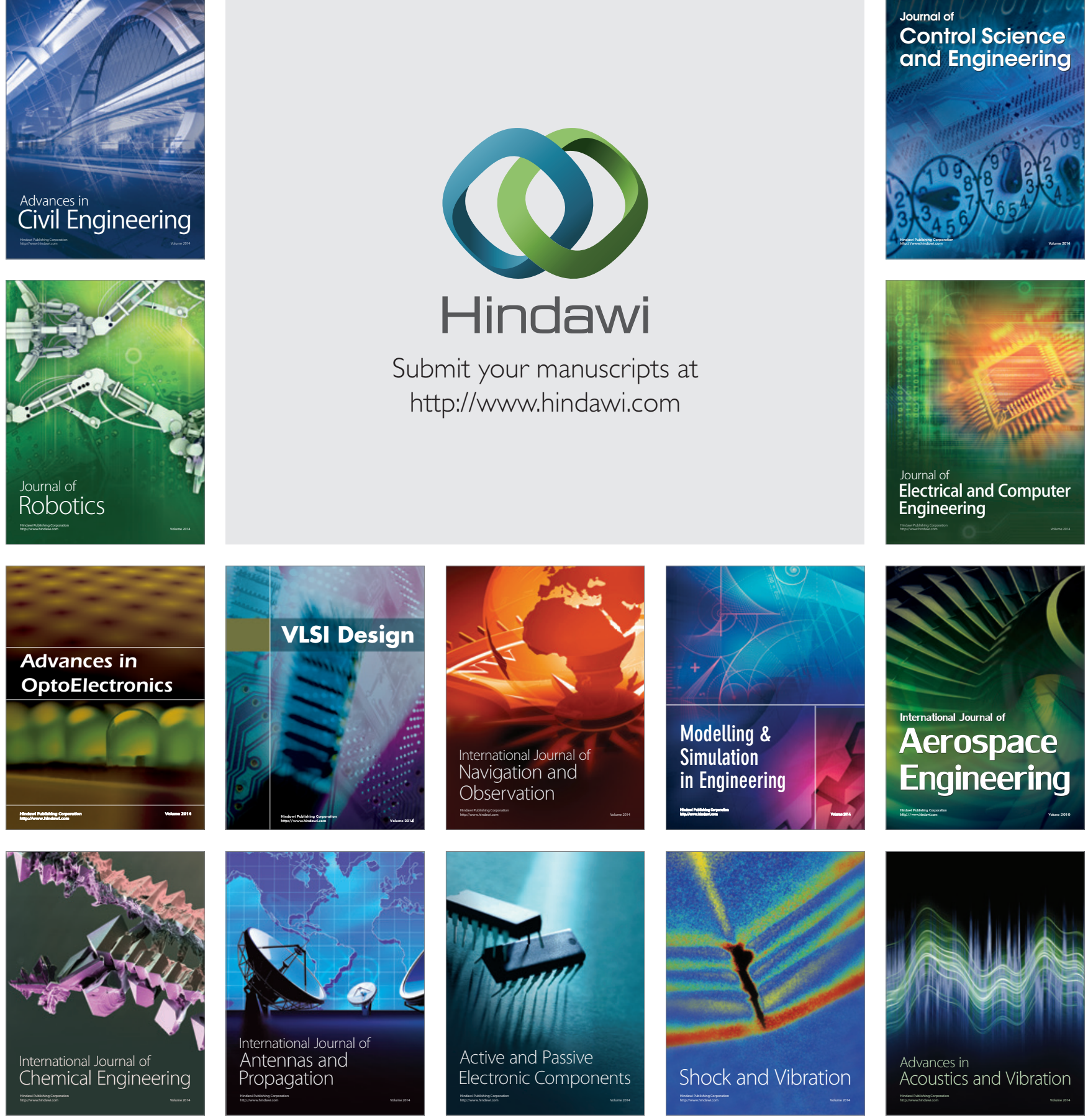